\title{
Reflexiones preliminares sobre el sistema de educación superior en Colombia y su papel en el contexto de un mundo globalizado
}

\author{
Preliminary reflections about the higher education system in Colombia and its role in the globalized world context
}

\author{
Maria Adela Villar Torres
}

\section{Resumen}

El proceso de globalización es un factor de orden económico que influyó en todas las dimensiones del desarrollo humano, con efectos positivos en unas y negativos en otras; una de estas dimensiones es la educativa, y es en particular en la educación superior donde se vislumbra la mayor incidencia.

Este articulo plantea sus reflexiones en dos periodos. El primer período aborda la relación entre globalización y educación superior, identifica algunas características del proceso de globalización e interpreta la educación superior desde lo planteado por la Unesco en su primera conferencia mundial. El segundo periodo corresponde a una caracterización preliminar del sistema de educación superior en Colombia, presenta una reseña histórica breve y un panorama de la década de 1990. En el desarrollo de cada aspecto se presentan razones especificas que determinan su elección. Concluye con algunas apreciaciones de lo que la autora considera puede ser el desafio de la educación superior en el nuevo milenio.

Palabras clave: Educación, educación superior, globalización, desarrollo.

\section{Abstract}

Key words: $\quad$ Education, higher education, globalization, development.

Fecha de recepción: 19 de septiembre de 2005.

Fecha de aceptación: 4 de noviembre de 2005.

Docente de la Facultad de Educación Física de la Universidad Pedagógica Nacional, Licenciada en Pedagogía y Psicologia de la Universidad Pedagógica Nacional, Especialista en Educación Superior y Pedagogia de la Universidad de San Buenaventura de Bogotá, en la actualidad desarrollando tesis para optar al título de Magister en Relaciones Internacionales de la Pontificia Universidad Javeriana. 


\section{Introducción}

Este documento hace parte de un trabajo de revisión documental y análisis bibliográfico iniciado por la autora hace dos años y busca dar cuenta del devenir de la educación superior en Colombia y de la incidencia que sobre ella ha tenido la globalización.

La revisión documental se realiza en tres momentos: el primero de ellos, al cual corresponde éste documento, pretende identificar dentro del contexto de globalización los nuevos retos para la educación superior colombiana; el segundo confronta al sistema educativo colombiano con los sistemas de educación superior de América Latina para identificar puntos en común y puntos de divergencia, lo cual se revisaron los casos de México y Chile; y el tercero reflexiona acerca del grado de coherencia entre las políticas educativas asumidas por el Estado colombiano para la educación superior y las necesidades de desarrollo que exige el país.

La revisión documental inicia su recorrido en la década de 1990, toda vez que contextualmente para Colombia con la Constitución Nacional de 1991 se orienta un nuevo derrotero, tanto para el país, como para la educación, y en particular, para la educación superior, con la Ley 30 de 1992.

\section{La globalización y la educación superior}

\section{La globalización y su contexto}

La globalización es un proceso de tipo económico, social y cultural que implica apertura de fronteras políticas, religiosas, étnicas, culturales y educativas; en este proceso - que se ha visto acelerado por los grandes avances en el área de las telecomunicaciones y la informática-, las relaciones establecidas entre modos de producción, el Estado y las formas de vida son abiertas. La globalización se produce dentro de un contexto de modernidad ${ }^{1}$, cuyas características se manifiestan en un énfasis en la racionalidad científica, el uso de tecnologías y la manipulación instrumental de las mismas. En este sentido, modernidad y globalización implican no sólo la modernización del aparato productivo, sino también la apertura a nuevos modelos sociales, la desacralización de la conciencia o cambio de actitud, la superación de dogmatismos o cambio de paradigmas, la apertura como cambio continuo en las formas de vida, la proyección de utopías como realidades y la búsqueda continua de la construcción de futuro (Sistema de Educación Superior; 1993: 13).
La globalización se ha manifestado de forma visible en las relaciones económicas, lo cual se vislumbra en el papel preponderante que han adquirido las empresas multinacionales y el comercio mundial, superando los límites geográficos de los Estados nacionales y afectando el desarrollo de sus pobladores en los ámbitos social y cultural. Como resultado de esto se han incrementado los niveles de pobreza en los países menos desarrollados, agudizando las cada vez más crecientes diferencias sociales y económicas.

Dados los efectos negativos de los procesos de apertura y de globalización que ha experimentado el mundo en los últimos años, la educación y sus responsables se ven abocados a asumir un rol acorde con las exigencias de la moderna sociedad del conocimiento. En la actualidad una economía logra integrarse eficientemente en el proceso de globalización en la medida en que desarrolle ventajas competitivas dinámicas ${ }^{2}$, ya que la competencia internacional no se da en el ámbito de las ventajas comparativas ${ }^{3}$, debido a que los recursos físicos son susceptibles de agotarse o de desarrollarse en otras latitudes ${ }^{4}$ y los costos relativos ventajosos en la producción dada la libre movilidad internacional de los recursos tienden a nivelarse; de modo que la única alternativa de lograr ventajas a largo plazo en el proceso de globalización es mediante la ampliación del acervo de capital humano y de capital social ${ }^{5}$. En este sentido, se hace indispensable la articulación entre el sistema de producción de conocimiento -la "escuela" ${ }^{3}$ y los medios masivos de comunicación-, y el sistema de producción de bienes y servicios -la industria-, pilares esenciales del proceso de globalización toda vez que el primero posibilitan el desarrollo y el fortalecimiento del segundo.

En particular, para Colombia las transformaciones del sistema educativo encaminadas a responder a dichas exigencias están reglamentadas por el Estado desde lo previsto en la Constitución Nacional, la Ley 30 de 1992 y la Ley 115 de 1994, que han transformado sus reflexiones y acciones (educación por competencias, evaluación por logros y acreditación de calidad, entre otros).

\section{La educación superior según la Unesco}

El 9 de octubre de 1998, la Organización para la Educación, la Ciencia y la Cultura de las Naciones Unidas proclama la primera declaración mundial sobre educación superior, a la que denominó: Declaración mundial sobre la educación superior en el siglo XXI: visión y acción, en la cual establece el marco de acción prioritario para el cambio y el desarrollo de la educación superior. En la 
declaración refiere, entre otros aspectos, que durante las últimas décadas la demanda mundial por acceder a la educación superior ha aumentado significativamente, no sólo en las áreas de formación tradicional, sino en una amplia gama de ofertas educativas que intentan responder -en algunos casos con relativo acierto- a las demandas del mundo globalizado.

Según sus datos, entre 1960 y 1995 la matrícula en las instituciones de educación superior se multiplicó en más de seis veces, pasando de 13 millones a más de 82 millones de estudiantes. Paralelo a este proceso de expansión en términos cuantitativos se ha dado un proceso de diferenciación y estratificación en cuanto a las oportunidades de acceso y las condiciones socioeconómicas de la población ${ }^{7}$. Este aspecto, aunque ha beneficiado a grupos poblacionales que no contaban con condiciones para acceder a la educación superior, ha contribuido a agudizar los problemas de calidad en los procesos de formación, ya que la principal afectada con dicha determinación ha sido la educación pública, que enfrenta problemas de hacinamiento, falta de recursos, disminución de la inversión e instalaciones insuficientes e inadecuadas para enfrentar la creciente demanda; a diferencia de la educación privada, que la ha asumido como una posibilidad de negocio, de comercialización del servicio educativo y de alto nivel de rentabilidad.

Por lo anterior, el principal desafío que enfrenta la educación superior (Declaración.... 1998) hoy es la ampliación de la oferta educativa respaldada por programas de calidad ${ }^{8}$; además, otros retos están relacionados con los problemas de financiación y democratización, y los procesos de cooperación internacional, en los cuales la educación y la formación profesional deben preocuparse porque los trabajadores tengan la oportunidad de adquirir las aptitudes que exige una economía en constante evolución.

En este contexto, la misión de la educación superior en torno de sus funciones básicas de formación, investigación y extensión debe contemplar los siguientes aspectos: garantizar igualdad en el acceso, el cual debe estar basado en los méritos, capacidades, esfuerzos y determinación de los educandos sin ningún tipo de discriminación, y facilitado con el desarrollo de nuevas modalidades de enseñanza como cursillos, estudios de tiempo parcial, cursos por módulos, educación a distancia, entre otros; fortalecer la investigación en todas las áreas del conocimiento y la difusión de sus resultados; desarrollar políticas a largo plazo cuya base de evaluación sea la concordancia entre lo que produce la universidad y lo que la sociedad espera de ella, de manera que se erradiquen progresivamente los problemas comunes a todas las sociedades, como la pobreza, la intolerancia, la violencia, el analfabetismo y el hambre; por último, desarrollar métodos educativos innovadores fundamentados en el pensamiento crítico y la creatividad (Ibid.: 16-23).

\section{La educación superior reflexionada a futuro}

En su declaración, la Unesco propone un conjunto de acciones prioritarias para el desarrollo de la educación superior a futuro, en tres ámbitos:

\section{Ámbito nacional}

El Estado y sus ramas constitutivas deberán crear el marco legislativo, político y financiero que reforme y desarrolle la educación superior, teniendo en cuenta su accesibilidad general en función del mérito, su estrecho vínculo con la investigación, el papel protagónico que deben asumir los estudiantes en la renovación de su educación y la promoción de la libre movilidad nacional e internacional de los actores del proceso educativo.

\section{Ámbito de los sistemas y las instituciones}

La misión de los establecimientos educativos deberá estar en relación con las necesidades presentes y futuras de la sociedad en que se desarrolla, por lo cual se hace necesario estrechar los vínculos entre la educación superior y el mundo del trabajo, lo que es particularmente importante en los países en desarrollo; así mismo, se debe crear instituciones de carácter técnico y apoyar a los graduados a través de créditos para la creación de pequeñas y medianas empresas, de modo que éstos no se conviertan en personas que sólo buscan trabajo, sino que sean generadores de empleo.

\section{Ámbito internacional}

Desarrollar proyectos de cooperación a través de organizaciones intergubernamentales y no gubernamentales entre los países y a través de proyectos de cooperación desde los países desarrollados hacia los países en desarrollo, basados en la solidaridad y la asociación. También se debe promover la movilidad universitaria internacional como medio de hacer progresar el saber. Este proceso de cooperación internacional busca garantizar el reconocimiento justo y razonable de los estudios cursados independientemente del lugar donde se realicen. Por último, es importante contrarrestar los efectos adversos de la fuga de cerebros y buscar retener al personal cualificado en sus países de origen?. 


\section{El sistema de educación superior colombiano}

\section{Evolución histórica}

Mirar de manera retrospectiva la educación superior en Colombia exige ubicarse en un mundo histórico y cronológico concreto. Aunque la universidad en el país se remonta a la época de la Colonia, con la llegada de las primeras comunidades religiosas que le confirieron desde entonces un carácter confesional, la constitución de la Universidad como una institución autónoma, estatal y laica, abierta a las diferentes corrientes de la ciencia y del saber moderno, corresponde al año 1935, año en el que fue reestructurada la Universidad Nacional de Colombia. Subsiguiente a lo anterior, en el país fueron desarrollándose procesos de expansión cuantitativa en cuanto a matrícula, instituciones y cuerpo docente; y de diferenciación cualitativa respecto al tipo de instituciones, programas y carreras, que marchan al compás de la modernización económica y de la concentración urbana, como ha sucedido en muchos lugares del mundo.

Además de las carreras tradicionales - derecho, medicina y filosofia-, se da el surgimiento de programas de corte moderno -aparición de ingenierías y ciencias económicas-, primero destinadas al consumo masivo y posteriormente, carreras largas o cortas, de tipo profesionalizante, con poco çontenido investigativo y de poca calidad.

La universidad tradicional, la universidad moderna y la universidad de masas se constituyen no sólo, como etapas históricas, sino superpuestas unas a otras hasta llegar a coexistir dentro del sistema y dentro de la misma institución.

Otros factores históricos permiten explicar ciertas características del sistema de educación superior colombiano haciendo referencia al contexto sociopolítico del país, propios de la manera en que se produjo el proceso de laicización del Estado y el carácter particular que revistió el proceso de urbanización, como la actuación misma de los actores que intervinieron en el sistema.

El proceso de laicización del Estado fue más tardío que en otros países de Latinoamérica. $\mathrm{Al}$ iniciarse el siglo XX el país era regido por la Constitución de 1886, la cual imprime un carácter "confesional" ${ }^{10}$ y otorga a la Iglesia Católica poder de control sobre el aparato educativo ${ }^{11}$. Durante los años treinta el Estado asume mayor protagonismo sobre el sector educativo cuando los liberales suplantan casi cinco décadas de hegemonía conservadora buscando acabar con el analfabetismo y brindando una educación mas popular ${ }^{12}$; sin embargo, la injerencia de la Iglesia en las políticas educativas y su autonomía sobre la educación privada se mantiene hasta la Constitución de $1991^{13}$; durante este periodo la Iglesia no perdió su poder político ni ideológico. Paralelamente a ello, la universidad moderna, estatal y laica que surge en la década de 1930 en vez de sustituir a la universidad confesional, pasó a convivir con ella, constituyéndose de esta manera el sistema de educación superior del presente siglo con dos sectores fuertes: uno, el estatal, compuesto por instituciones nacionales y regionales, y el otro, el privado, dominado inicialmente por órdenes religiosas y más tarde por instituciones no confesionales de derecho privado (Lucio y Serrano: 1993).

El proceso de concentración urbana se inicia en la década de 1950, se acelera en las décadas siguientes con un crecimiento del 5,6\% anual, y empieza a desacelerarse a partir de la década de 1980 frente a los parámetros de las naciones en vía de desarrollo gracias a un fenómeno de transición demográfica. En la década de 1960, durante el Frente Nacional, se presenta la confrontación de posiciones provenientes de diversos actores sociales y políticos en torno a la educación superior; los analistas las agrupan en dos líneas fundamentales: la posición desarrollista, que buscaba modernizar la universidad para hacer de ella un instrumento eficiente al servicio del capital, y la posición contestataria, que fluctuaban desde posiciones reformistas hasta posiciones radicales. En las dos décadas posteriores se presenta una expansión del sistema educativo, incrementándose el valor en las matrículas y el número de las instituciones, confrontado con un decrecimiento de la población estudiantil.

\section{Panorama de la década de 1990}

El punto de partida de la caracterización educativa de la década de 1990 se inicia con el Decreto 80 de 1980, mediante el cual se reestructura la educación superior, y se consolida con la Ley 30 de 1992. Durante este período surge un conjunto heterogéneo y disperso de instituciones medianas y pequeñas en las que se entrecruzan distintos actores: por un lado, entidades estatales regidas por la nación, la región o el municipio; y por el otro, entidades de carácter privado regidas por comunidades religiosas, corporaciones privadas o grupos de carácter político y/o económico.

Al no haber políticas estatales claras que organizaran el sistema, se añade a ello el deterioro y desprestigio de la universidad estatal. Para el Estado y ciertos sectores influyentes de la opinión pública, la universidad oficial se había convertido en potencial de problemas de orden público que había que reprimir, lo cual produjo el cierre de varias universidades oficiales. 
A partir de 1980 el Estado busca retomar la iniciativa en materia de educación superior, mediante tres tipos de medidas orientadas a:

\section{Estructurar y organizar el sistema}

La pieza clave es el Decreto 80, que busca organizar la educación superior, definiendo primero sus componentes para luego estructurarlos como sistema. Como componentes básicos se establecen cuatro modalidades posibles de educación superior: dos no universitarias y dos universitarias: 1. La formación intermedia profesional, más tarde denominada formación técnica. 2. La formación tecnológica. 3. La formación universitaria propiamente dicha, y 4. La formación avanzada, en sus modalidades de especialización, magíster y doctorado (Ibíd).

Al estructurar el sistema se pretende, en primer lugar, dar posibilidad de flujo entre las modalidades; en segundo lugar, se jerarquizan las instituciones de acuerdo con las modalidades que están facultadas para ofrecer; en tercer lugar, el Decreto 80 configura el sistema con base en un marco legislativo unificado para cada uno de los sectores: oficial y privado.

\section{Fomentar los procesos de expansión y democratización}

Debido a la gran permisividad del Estado en la década de 1980 , la definición laxa de universidad ${ }^{14}$, hize que se admitieran como tales muchas instituciones pequeñas con dudoso nivel de calidad. Aún así, la política estatal más clara destinada a estimular la expansión del sistema se dio en este tiempo con la promoción de la educación superior a distancia entre 1982 y 1986, mediante la creación de la Universidad Nacional Abierta y a Distancia, UNAD.

\section{Mejorar la calidad del sistema}

Como consecuencia de la expansión de la Universidad, el modelo de universidad de masas ${ }^{15}$ incorporó en el desarrollo de su práctica formativa la función de investigación $^{16}$. Para contrarrestar la baja calidad imperante en su momento, el gobierno emprendió iniciativas como la consolidación de un programa nacional de ciencia y tecnología, el fortalecimiento de los programas de posgrado $y$, con el apoyo del Icfes, se puso un límite a la proliferación de programas e instituciones de baja calidad ${ }^{17}$.

\section{Características del sistema de educación superior colombiano}

En el estudio realizado por la misión de ciencia, educación y desarrollo se plantea una reflexión sobre la educación superior en Colombia desde su problemática y desde los núcleos que la comprometen. Como principa- les problemas del sistema de educación superior colombiano se plantean:

a. Bajacalidad.

b. Escasa investigación, incluso en los programas de posgrado.

c. Énfasis profesionalizante en algunas universidades, modelo ventajoso porque exige menores niveles de inversión en los ámbitos científico e investigativo. Así, el proceso de formación se basa en la transmisión del conocimiento con poca producción del mismo, dando como resultado profesionales desactualizados e incapaces de actuar de manera productiva, para satisfacer las necesidades sociales y culturales del país.

d. Ausencia de una cultura académica, donde los mecanismos de crítica científica apenas están consolidándose.

e. Las instituciones de educación superior en Colombia no conforman un sistema relacionado con los niveles anteriores de la educación (preescolar, primaria y secundaria), donde se planteen objetivos comunes. La universidad se mantiene aislada administrativa y socialmente del contexto en el que ejerce; asimismo, las posibilidades de acceso por parte de quienes terminan la educación media es escasa y a su vez no hay una atención equitativa a quienes ingresan a la educación superior.

f. Con la Ley 30 se ha generado una desordenada y veloz expansión cuantitativa de los programas de educación superior, generando masificación, disminución del tiempo efectivo de estudio y de las exigencias académicas for males; sumado a la excesiva diversificación de programas de pregrado ${ }^{18}$, especializaciones y maestrías.

g. Las interrelaciones pedagógicas, curriculares y extracurriculares entre docentes y estudiantes están desapareciendo. En consecuencia, la calidad institucional, académica y ético-política se ha visto afectada y con ella la incapacidad de formar una cultura académica y consolidar una dirigencia visionaria, ética, crítica y autocrítica.

h. La calidad educativa es disímil según la institución; estudios demuestran que las tasas de desempleo profesional y los niveles salariales de los egresados son muy diferentes según la universidad que expida el título profesional.

i. Las características señaladas antes conducen a una deficiencia en la gestión administrativa tanto en la universidad pública como en la privada. Nuestra cultura universitaria da prioridad a la administración y no a la academia. 
Desde la problemática por núcleos se plantea:

\section{Calidad de la educación}

a. Pobreza en la conceptualización. Se insiste en ampliar la cobertura sin mejorar la calidad. Diversas experiencias han demostrado que la expansión cuantitativa sin un acompañamiento de esfuerzos por mejorar la calidad genera mayores tasas de deserción, repitencia y aprendizajes muy bajos.

b. Predomino de la instrucción. La enseñanza entendida como una instrucción rígida, metódica y orientada a los aprendizajes observables; no enfatiza en la construcción del conocimiento.

c. Descuido en la formación de valores y principios. El sistema educativo tiene poca incidencia en la formación de valores y actitudes ciudadanos y democráticos, lo cual incide sobre la calidad del desarrollo profesional y sobre el desempeño ético de los profesionales frente al campo laboral.

\section{Políticas estatales}

a. Predominio de políticas focalizadas en aspectos específicos.La gestión dirigida hacia aspectos puntuales de la educación descuida la visión global del sistema y su problemática.

b. Políticas centralizadas. Por lo general las políticas se planean y diseñan desde los sectores centrales de la organización educativa, casi siempre tienen el carácter de normas, leyes o regulaciones que suponen la aceptación y cumplimiento por parte de las instancias locales, desconociendo la diversidad regional y cultural del país y la complejidad del sistema escolar.

c. Políticas desinformadas. Se carece de un sistema de información que sirva de soporte para orientar y dar a conocer las políticas estatales. Se cuenta con un conjunto de evaluaciones descriptivas con problemas de validez y de confiabilidad en los datos. Faltan evaluaciones de tipo causal y, en especial, sobre el impacto real causado por las reformas iniciadas.

d. Políticas y gestiones discontinuas. Son el principal problema de las políticas educativas, pues su formulación e implementación dependen de los gobiernos de turno y de los intereses políticos particulares de cada administración.

\section{La educación superior en Colombia para el nuevo milenio}

Algunos atribuyen la baja calidad en la educación a la falta de un sistema de control y de fomento estatal. Otros se resisten a una presencia excesiva del Estado y abogan por un sistema de educación más autónomo, con instituciones que decidan por sí mismas el tipo de servicios que han de prestar, su contenido, así como el costo del servicio educativo definido según las leyes del mercado (demanda y oferta).

Ante un panorama universitario segmentado y diferenciado, un importante reto para la sociedad está en abrir canales de acceso a las universidades de calidad a través de mecanismos como becas y créditos, de forma que el filtro se dé a partir de los méritos académicos y formativos en lugar del estrato socio-económico.

Además, y siguiendo las recomendaciones sugeridas por la misión de ciencia y tecnología, es importante:

\section{Cualificar el sistema escolar a través de:}

a. Priorizar a la calidad en la educación en cuanto a insumos, agentes, procesos, ambientes y productos en todos los niveles educativos.

b. Fortalecer el sistema nacional de evaluación de la educación, para que esté en capacidad de hacer estudios teóricos, investigativos y evaluativos, y pueda divulgar y someter a discusión la información obtenida.

c. Renovar la educación superior fomentando la generación de conocimiento dentro de la universidad. Ello contribuye a que la universidad asuma la generación de conocimiento nuevo a través de la investigación de problemas sociales relevantes, y universalmente nuevo a través de investigaciones de frontera; a conformar equipos de investigadores que cuenten con la mejor dotación, instrumentos de laboratorio, bibliotecas, sistemas de información y recursos apropiados para alcanzar la excelencia; a adelantar programas de doctorado asociados a un sistema integrado de institutos y programas de investigación de excelencia.

d. Recomienda: desarrollar estrategias y acciones de apoyo para quienes presenten mayores probabilidades de deserción; diseñar ciclos de formación en los que se otorguen títulos intermedios en aquellas carreras en las cuales su estructura se preste para ello; incorporar el número de docentes de tiempo completo comprometidos con la creación de conocimiento y la promoción de sus estudiantes; aumentar la capacidad de respuesta a las demandas sociales, en particular aquellas de servicios puntuales y de soluciones a corto plazo mediante programas de extensión; crear institutos universitarios universitarios de alto nivel académico con adecuada infraestructura y dotación suficiente para desarrollar carreras de acuerdo con 
la exigencia del proceso de modernización; generar mecanismos para la formación extracurricular en los que estudiantes de niveles avanzados se vinculen temporalmente a entidades de carácter nacional y desarrollen trabajos de investigación de interés social; $y$ por último, articular la educación básica y media con la superior.

\section{Conclusiones}

1. Se hace indispensable concienciar en cuanto a que la educación es una inversión y no un gasto, para que el Estado, la familia y la empresa pública y privada apoyen reformas educativas que redunden en beneficio social.

2. Los costos de la educación deben considerarse desde el punto de vista de sus productos sociales a largo plazo, ya que el proceso educativo genera un sinnúmero de externalidades positivas que superan los costos directos generados en el proceso ${ }^{19}$.

3. La preocupación del gobierno nacional debe ir más allá de garantizar el acceso masivo de la población a los niveles de educación básica. Éste es, sin duda, un paso importante en la búsqueda de escolarizar masivamente a la población colombiana y de sentar bases para un desarrollo económico sostenible.

No obstante, éste es sólo el primer peldaño de una estrategia de política educativa más amplia y más acorde con las actuales exigencias de un mundo globalizado.

4. El logro de una escolarización a todo nivel (básica, media, técnica, tecnológica y superior) generalizada en nuestro país requiere importantes esfuerzos de los sectores público y privado para ampliar la oferta educativa con un respaldo en términos de calidad, y para que esa mayor oferta esté acompañada de una demanda efectiva ${ }^{20}$.

5. El compromiso del Estado está en ampliar la capacidad instalada, aumentando las Universidades públicas y fortaleciendo la capacidad de las existentes a través de mecanismos para el incremento del presupuesto de inversión.

Así mismo, el Estado debe retomar el enfoque tradicional de subsidiar la oferta del servicio educativo y no privatizar éste ${ }^{21}$ garantizando su gratuidad, y a la vez subsidiando la demanda en casos puntuales ${ }^{22}$. Una opción que contribuye con el proceso de financiamiento de la educación superior consiste en que los niveles más avanzados, como maestría y doctorado, colaboren con la financiación del pregrado, de forma que los costos más altos por matrícula estén a cargo de estos niveles, mientras que el pregrado sea gratuito.

6. El compromiso del sector privado está también en ampliar la oferta educativa, pero no de forma indiscriminada como lo ha permitido la Ley 30 , sino con programas de calidad. En este sentido, es importante que las universidades acrediten sus programas y que los requisitos exigidos para tal fin sean rigurosos.

7. La universidad colombiana debe involucrarse con la situación económica, social y política por la que atraviesa el país; del interior de los establecimientos educativos deben surgir las propuestas y las soluciones para superar la crisis. A través del cumplimiento de las funciones la universidad puede impactar los ámbitos local, regional y nacional.
AHUMADA, Consuelo. (1996). El modelo neoliberal y su impacto en la sociedad colombiana. Bogotá. Áncora Editores.

Cooperativa Editorial Magisterio. (1995). Código Educativo I. Bogotá. Serie Documentos No 12.

Código Educativo III. (1996). Bogotá. Serie Documentos No. 17.

COX, Cristian. (1996). Politicas de educación superior. En Politicas Comparadas de educación superior en América Latina. Chile. Flacso.

EL SISTEMA DE LA EDUCACIÓN SUPERIOR Y LA MODERNIDAD. (1993). Consigna, número 436. pág. 13. vol. XVII, abril - junio.

GÓMEZ MARTÍNEZ, Alberto. (1993). Reformas en curso de la educación colombiana. En El Acontista, año 1. Marzo. Fundación Saeta.

GOMEZ, Víctor y otros. (1998). Democracia y productividad. desafios de una nueva educación media en América Latina. Bogotá. Editorial Magisterio.
KENNEDY, Paúl. (1994). Hacia el siglo XXI: Un exhaustivo análisis de las fuerzas y tendencias que perfilarán el nuevo siglo. Plaza y Janes Editores.

LE BOT, Ivoón. (1985). Educación e ideologia en Colombia. Bogotá. Editorial La Carreta.

LUCIO, Ricardo, SERRANO, Mariana. (1993). La educación superior en Colombia: politicas estatales. En Politicas comparadas de educación superior en América Latina. Flacso.

MEJÍA, Marco Raúl. (1996). Educación y escuela en el fin de siglo. Bogotá. Cinep.

MISAS, Gabriel. (1999). El Plan de Desarrollo y las Políticas para la Educación Superior. En Cuadernos de Economia, No. 30. Bogotá. Universidad Nacional de Colombia.

(1993). El sistema de educación superior y la modernidad. En Revista Consigna. No. 436, Vol. XVII, abril -junio.

MISIÓN DE CIENCIA, EDUCACIÓN Y DESARROLLO. (1995). Colombia al filo de la oportunidad. Bogotá. Editorial Magisterio. 
MOSQUERA MESA, Ricardo. (1992). Concepción de la Reforma de Educación Superior. En Memorias del Seminario sobre ley 30 de Bogotá, Universidad de los Andes y Universidad Nacional de Colombia.

SERNA, Rollin Kent. (1993). El desarrollo de políticas en educación superior en México. En Políticas comparadas de educación superior en América Latina. México. Flacso.
TOFFLER, Alvin. (1994). El cambio del poder. Barcelona (España). Plaza y Janes Editores.

UNESCO, (1998). Conferencia Mundial sobre la Educación Superior: la Educación Superior en el Siglo XXI: Visión y Acción. 9 de octubre. Barcelona. www.unesco.org/education/educprog/wche/ declaration_spa.
Notas

1 Aunque existe una gran diversidad de posturas en cuanto a lo que los expertos consideran como modernidad, sus características y ubicación histórica; para efectos del presente artículo se entiende como el momento histórico, político y social (finalización del periodo de guerra fria, caida del muro de Berlín, desaparición del modelo comunista, perestroika) enmarcado por un modelo de desarrollo capitalista, neoliberal y del cual su última fase corresponde a la globalización.

2 Capacitación del recurso humano, desarrollo de comunidades académicas, procesos de investigación, etc.

${ }^{3}$ Disponibilidad de recursos, costos relativos, etc.

4 Téngase en cuenta la tendencia hacia la internacionalización de la mano de obra, la cual se contrata y se realiza en el país donde los costos sean más favorables para el fabricante.

5 La ampliación del acervo de capital humano se logra a través de procesos de capacitación, desarrollo de destrezas y habilidades, ampliación de la cobertura y calidad de la educación de la población, mientras que la ampliación del acervo de capital social implica el desarrollo de las condiciones propicias del entorno que permita el mejoramiento de las relaciones entre los individuos y las instituciones, para favorecer el crecimiento de las economías.

6 Entiéndaserpor "escuela" todo el sistema educativo nacional formal y no formal, y los espacios y momentos que hacen posible la adquisición de conocimiento (educación informal).

7 Diferenciación y estratificación mediante mayores oportunidades para los estratos 1 y 2 , disminuyendo los niveles de exigencia y rigurosidad en los procesos de selección.

${ }^{8}$ Calidad en función de cualificación de docentes, una nueva formación basada en competencias, ampliación de la investigación y extensión universitaria, pertinencia de currículos, generación de perfiles de oferta laboral acordes con la demanda de empleo, entre otros.

9 Vale la pena resaltar el efecto adverso que tiene la fuga de cerebros para los paises de origen al generar un desbalance a favor de los paises receptores quienes, en la mayoria de casos, no realiza la inversión requerida para la cualificación de ese recurso humano, pero sí reciben sus beneficios. En términos de externalidades, éstas son negativas en los paises de origen y positivas en los paises receptores.

${ }^{10} \mathrm{El}$ sólo preámbulo de la constitución modificado mediante plebiscito en 1957 determina: "En nombre de Dios, fuente suprema de toda autoridad, y con el fin de afianzar la unidad nacional, una de cuyas bases es el reconocimiento hecho por los partidos políticos de que la religión católica, apostólica y romana es la de la nación, y que como tal, los poderes públicos la protegerán y harán que sea respetada como esencial elemento de orden social, y para asegurar los bienes de la justicia, la libertad y la paz, el pueblo colombiano, en plebiscito nacional decreta..." (Henao Hadrón Javier. Constitución Política de Colombia. Editorial Temis. Bogotá. 1990).
${ }^{11}$ Mediante la firma del concordato de 1887 que le otorga total autonomia a la iglesia para impartir educación en Colombia. Establece como obligatoria la enseñanza religiosa en colegios, escuelas y universidades; y permite la expansión de la educación privada mediante la llegada al pais de nuevas órdenes religiosas (Maristas y Salesianos).

${ }^{12} \mathrm{Al}$ pais llegan modelos educativos europeos como el modelo Decroly con Agustín Nieto Caballero y la incidencia de la misión alemana en 1924, 1947 y 1952.

${ }^{13}$ A diferencia de la Constitución de 1886, la de 1991 determina la libertad de cultos y la pluralidad, $\mathrm{y}$ asume total control sobre el aparato educativo regulando su inspección y vigilancia.

${ }^{14}$ Presencia de tres programas académicos y existencia de capacidad investigativa

${ }^{15} \mathrm{El}$ concepto responde a las políticas de cobertura, ya que el porcentaje de estudiantes que logran acceder a la educación superior es mínimo en comparación con el número de estudiantes que terminan su educación secundaria.

${ }^{16}$ Función que busca entre otras cosas que la oferta educativa responda a las _necesidades sociales y laborales

${ }^{17}$ A esta necesidad responden las politicas de acreditación de calidad

${ }^{18}$ Con mayor tendencia mercantilista que académica. Algunas instituciones ofrecen programas para los cuales no cuentan con la infraestructura mínima (física, técnica, humana) necesaria que brinde una formación confiable y de calidad.

${ }^{19}$ Algunas externalidades positivas del proceso educativo son las investigaciones, la acumulación de competencias en docentes e investigadores, aportes a la formación de una cultura ciudadana, entre otras. Éstas se traducen en beneficios para el conjunto de la sociedad, de los cuales no se apropia sino marginalmente el estudiante.

${ }^{20}$ Que la sociedad tenga los recursos y las condiciones de vida que les permita educarse.

${ }^{21}$ Servicio que es un derecho fundamental del individuo.

${ }^{22}$ Actualmente se está dando un tránsito de los subsidios a la oferta hacia los subsidios a la demanda. Esto implica que se privatizan las universidades públicas y se otorgan créditos a través de instituciones financieras como el Icetex para que los estudiantes costeen su matricula. Esta forma de subsidio reduce la población beneficiada por el subsidio educativo y, además, no favorece a quienes verdaderamente necesitan de esos créditos. En algunos paises desarrollados, como Francia, Alemania, Italia, Estados Unidos e Inglaterra, el costo de la educación superior pública es nulo, y cuando se presentan subsidios a la demanda son subsidios para la manutención de los estudiantes. 